INVESTIGACIÓN ORIGINAL

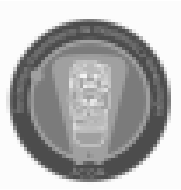

\title{
FACTORES ASOCIADOS AL PAPILOMAVIRUS HUMANO EN MUJERES MEXICANAS
}

María del R. González-Losa, MD* Gerardo Polanco-Marín, QFB*, Marylin_Puerto-Solís, MC*, Pedro Murguía-Mesina $\mathbf{M C}^{\dagger}$

Recibido: febrero 14/2002 - Revisado: abril 5/2002 - Aceptado: junio 24/2002

\section{RESUMEN}

El objetivo de este estudio es determinar las variables epidemiológicas asociadas a la infección por papilomavirus humano en un grupo de mujeres mexicanas. En un estudio prospectivo, transversal y comparativo, se estudiaron 470 mujeres que acudieron para valoración citológica y colposcópica; previo al examen ginecológico se les realizó una entrevista en la cual se consignó la ficha de identidad, historia reproductiva, anticonceptiva, sexual, tabaquismo pasivo y activo. El diagnóstico de papilomavirus se realizó por citología y colposcopia. Las mujeres infectadas fueron más jóvenes y tuvieron menos embarazos y partos que las mujeres sanas. En el análisis multivariado la edad de las pacientes y la edad del primer embarazo resultaron ser factores protectores contra la infección. A diferencia de otras investigaciones, este estudio no encontró ninguna asociación entre la infección con papilomavirus y la conducta sexual.

* Laboratorio de Virología, Centro de Investigaciones Regionales, Dr. Hideyo Noguchi, Universidad Autónoma de Yucatán, Mérida, México

MC. Clínica de Displasias, Hospital General "Carlos Ortiz Mariote”, Secretaría de Salud, Colima, México.
Palabras clave: papilomavirus humanos, colposcopia; factores de riesgo.

\section{SUMMARY}

The purpose of the study is to determine the epidemiological variables associated to human papillomavirus infection in a group of Mexican women. A cross-sectional study was conducted in 470 women enrolled for Pap smear and colposcopy. Previous to gynecological examination women were interviewed about demographic data, reproductive and contraceptive history, sexual behaviour and smoking. The diagnosis of papillomavirus was done by cytology and colposcopy. Women infected were younger and had less pregnancies and childbirth than healthy women. In a multivariate logistic regression analysis, the age of patients and the age of their first pregnancy were protective factors against the infection. Unlike previous research, this study found no associations between human papillomavirus and sexual behaviour.

Key words: human papillomavirus, colposcopy, risk factors. 


\section{INTRODUCCIÓN}

El cáncer cervicouterino (CACU) es uno de los principales problemas de salud pública en el ámbito mundial. Anualmente se diagnostican $500.000 \mathrm{ca}-$ sos nuevos, de los cuales $80 \%$ corresponden a los países en vías de desarrollo (1). En Latinoamérica se presentan cada año 68.000 casos nuevos. A lo largo de las tres últimas décadas, la mortalidad por cáncer cervicouterino en los países industrializados ha disminuido en forma sostenida, no así en los países en vías desarrollo, donde se ha mantenido estable. En un estudio realizado en Latinoamérica se encontró que Cuba, México y el Caribe tienen las mayores tasas de mortalidad por CACU (2).

En México el CACU es la primera causa de muerte por neoplasia en mujeres de vida reproductiva, a pesar de los esfuerzos realizados por la Secretaría de Salud, ya que desde 1974 existe un programa de detección oportuna de cáncer cervicouterino, el cual es nacional y gratuito (3).

Existen suficientes evidencias epidemiológicas y experimentales para afirmar que la infección del tracto genital femenino por algunos tipos del papilomavirus humano $(\mathrm{PVH})$ es el principal factor de riesgo para desarrollar cáncer cervicouterino (47). Se considera que la infección del tracto genital femenino por este virus es la infección de transmisión sexual más común $(8,9)$.

Existen más de 100 genotipos del PVH identificados plenamente, $\mathrm{y}$ de estos, 30 se han encontrado en el tracto genital femenino, de los cuales los genotipos 16, 18, 45, 31, 33, 35, 39, 45, 51, 52, 56, $58,59,66$ y 68 han sido asociados con mayor frecuencia a CACU, por lo cual se les denomina de "alto riesgo" (10).

En los últimos años se han realizado estudios para conocer cuáles son los factores de riesgo para la infección por este virus; sin embargo, los resultados han sido variables y no siempre consistentes, y sugieren que existen diferencias poblacionales en los factores que predisponen a la infección por VPH (11-13).
En México se ha podido determinar que la infección por PVH16/18 es un factor de riesgo independiente para desarrollar CACU (14).

El objetivo de este estudio es determinar las variables epidemiológicas asociadas a la infección por virus de papiloma humano en un grupo de mujeres mexicanas.

\section{MATERIALES Y MÉTODOS}

Se realizó un estudio prospectivo, transversal y comparativo durante el periodo comprendido entre diciembre de 1996 y octubre de 1997.

Se estudiaron de manera consecutiva 470 mujeres que acudieron para valoración citológica y colposcópica al Hospital General "Carlos Ortiz Mariote" de la Secretaría de Salud en la ciudad de Colima, México.

Previo al examen ginecológico, sin conocer los resultados del mismo, se les realizó una entrevista por una trabajadora social, en la cual se consignaron la ficha de identidad y la historia reproductiva (edad de menarquia, número de gestaciones, número de partos, número de abortos y cesáreas, edad de primer parto), anticonceptiva, sexual (inicio de vida sexual y número de compañeros sexuales) y de tabaquismo pasivo y activo.

Aquellas mujeres cuyo diagnóstico citológico y colposcópico fue de displasia en cualquier grado fueron excluidas del estudio.

El diagnóstico de infección por PVH se realizó por medio de citología y colposcopia; esta última fue realizada por un médico ginecólogo y colposcopista. La asociación entre las variables epidemiológicas y la presencia de PVH se analizó por medio de $\mathrm{X}^{2}$ (con un intervalo de confianza de $95 \%, \mathrm{p}<0,05)$ y T Students.

Posteriormente, para determinar la independencia de los factores asociados se realizó regresión logística, utilizando el STATA 5,0. 


\section{RESULTADOS}

De las 470 pacientes estudiadas, 154 (32,76\%) fueron positivas a IPVH y 316 (67,23\%) negativas. En cuanto a la distribución por edades de la infección por PVH, la máxima frecuencia se encuentra en mujeres menores de 25 años y disminuye progresivamente (Figura 1).

Al realizar la comparación de medias de las variables cuantitativas por medio de la T de Students, se observó una diferencia estadística entre ambos grupos en tres variables: edad de las pacientes, número de gestaciones y número de partos.

La media para la edad fue la siguiente: positivas 31,6 años; negativas 35,0 años. Para el número de gestaciones la media para el grupo con IPVH fue 2,3 y para el grupo de mujeres sanas 3,2. La media de partos para las mujeres con infección fue 2,6 y para las mujeres negativas 3,3. Las mujeres con IPVH fueron significativamente más jóvenes y con una gestación y un parto menos que las mujeres sanas.

Con relación a las otras variables cuantitativas estudiadas se encontró similitud en ambos grupos y no hubo una diferencia estadísticamente significativa; la media de la edad de la menarquia para ambos grupos fue de 13 años.

La media de la edad de inicio de vida sexual fue 18 años para ambos grupos. La edad del primer embarazo fue ligeramente menor en el grupo de positivas $(18,6$ años) que en el de las mujeres sanas $(19,26$ años). La media del número de cesáreas fue igual para ambos grupos $(0,3)$. La media del número de abortos fue 0,31 y 0,45 para positivas y negativas respectivamente. En cuanto al número de compañeros sexuales, $77,87 \%$ de todas las mujeres refirió haber tenido sólo uno durante su vida, 14,25\% dos, y sólo $7,87 \%$ contestaron haber tenido tres o más, siendo la media de 1 para ambos grupos. (Figuras 2 y 3).

Al realizar ji cuadrado y cálculo de odds ratio crudo de las variables cualitativas, no se encontró ninguna diferencia entre ambos grupos (Tabla 1).
El análisis multivariado se ajustó por edad, edad de primer embarazo, número de partos, número de cesáreas y de compañeros sexuales, y se pudo observar que al aumentar la edad de las pacientes

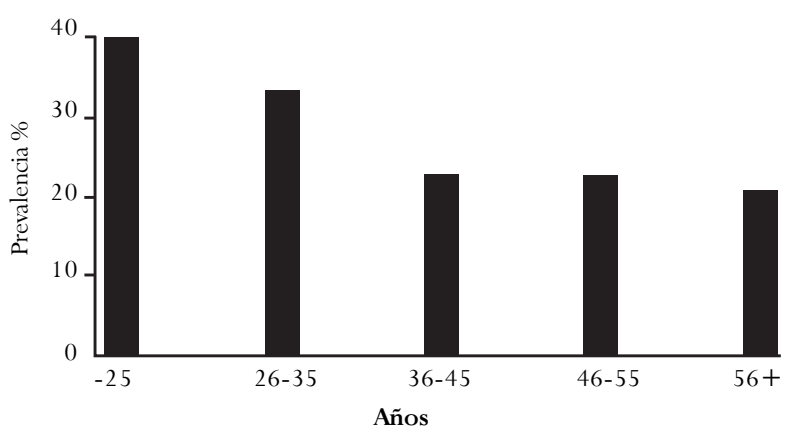

Figura 1. Distribución de papilomavirus humano por grupos de edad.

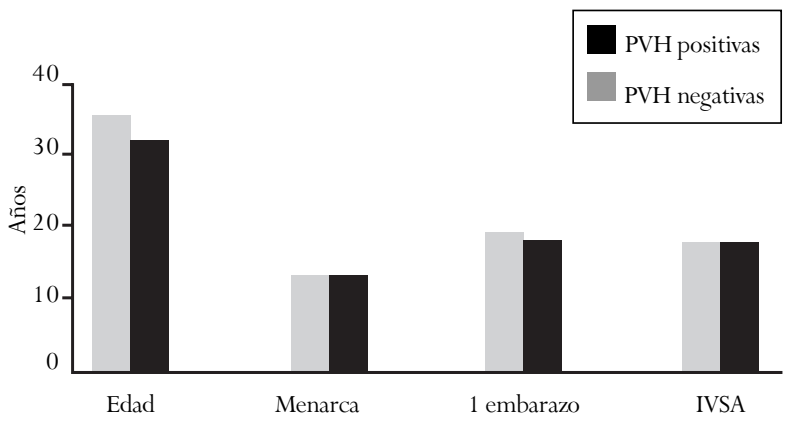

Figura 2. Características epidemiológicas de mujeres PVH positivas y PVH negativas.

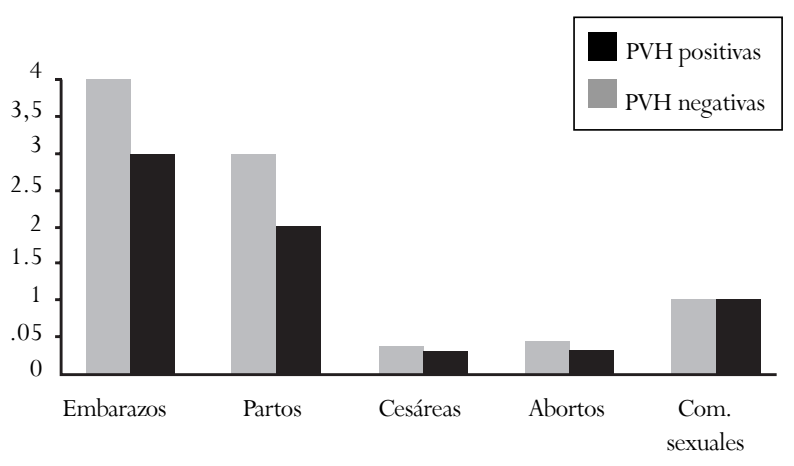

Figura 3. Características epidemiológicas de mujeres PVH positivas y PVH negaticvas. 


\begin{tabular}{|c|c|c|c|c|c|c|}
\hline \multicolumn{7}{|c|}{$\begin{array}{l}\text { TABLA 1. FACTORES DE RIESGO ASOCIADOS A IPVH: } \\
\text { ANTICONCEPTIVOS Y TABAQUISMO }\end{array}$} \\
\hline Factor & PVH positivas & PVH negativas & $\mathbf{O R}^{1}$ & 95\% IC & $\mathrm{OR}^{2}$ & 95\% IC \\
\hline \multicolumn{7}{|c|}{ Tabaquismo } \\
\hline No & 140 & 289 & 1,00 & & 1,00 & \\
\hline Sí & 14 & 27 & 1,07 & $0,52-2,20$ & 1,03 & $0,50-2,10$ \\
\hline \multicolumn{7}{|c|}{$\begin{array}{l}\text { Tabaquismo } \\
\text { pasivo }\end{array}$} \\
\hline No & 106 & 210 & 1,00 & & 1,00 & \\
\hline Sí & 57 & 97 & 1,16 & $0,76-1,77$ & 1,23 & $0,81-1,87$ \\
\hline \multicolumn{7}{|l|}{ AO } \\
\hline No & 125 & 257 & 1,00 & & 1,00 & \\
\hline Sí & 29 & 59 & 1,01 & $0,60-1,70$ & 0,95 & $0,57-1,59$ \\
\hline \multicolumn{7}{|l|}{$\mathbf{A P}$} \\
\hline No & 135 & 280 & 1,00 & & 1,00 & \\
\hline Sí & 19 & 36 & 1,09 & $0,58-2,05$ & 0,95 & $0,51-1,76$ \\
\hline \multicolumn{7}{|l|}{ DIU } \\
\hline No & 96 & 219 & 1,00 & & 1,00 & \\
\hline Sí & 58 & 97 & 1,36 & $0,89-2,06$ & 1,16 & $0,75-1,81$ \\
\hline
\end{tabular}

DIU: dispositivo intrauterino. AO: anticonceptivos orales. AP: anticonceptivos parenterales. OR ${ }^{1}$ : Odds ratio crudo. OR ${ }^{2}$ : Odds ratios ajustada por: edad, edad de primer embarazo, número de partos, número de cesáreas y número de parejas sexuales.

disminuía el riesgo de enfermedad, de manera que en las mujeres mayores de 25 años se encontró un odds ratio de 0,59 e IC95 (0,36-0,97), el cual disminuyó progresivamente al aumentar la edad.

En cuanto a la edad del primer embarazo, se pudo observar una relación inversa entre la edad y el riesgo, de manera que para aquellas mujeres que se embarazaron por primera vez después de los 23 años se estimó una razón de momios de 0,49 e IC95 $(0,25-0,97)$ (Tabla 2).

\section{DISCUSIÓN}

Estudios previos para determinar los factores asociados a la infección por PVH han mostrado resultados inconsistentes y a veces contradictorios; sin embargo, un hecho importante es que se han encontrado diferencias significativa en cuanto al patrón de factores de riesgo según la población estudiada $(15,16)$.

En la presente investigación, cuando se realizó el análisis univariado, se encontró que en las variables: edad de la paciente, número de partos y número de gestaciones había una diferencia estadísticamente significativa entre ambos grupos, pues las mujeres del grupo de casos eran más jóvenes y, en promedio, tenían una gestación y un parto menos que las mujeres sanas. Más adelante, al realizar el análisis multivariado, sólo la variable edad continuó siendo el factor asociado IPVH. 
TABla 2. FaCtores DE RIESGo ASOCIADOS A IPVH: ANÁLISIS MULTIVARIADo

\begin{tabular}{|c|c|c|c|c|c|c|c|}
\hline Factor & $\begin{array}{c}\text { PVH } \\
\text { positivas }\end{array}$ & $\begin{array}{c}\text { PVH } \\
\text { negativas }\end{array}$ & $\mathrm{OR}^{1}$ & 95\% IC & $\mathrm{OR}^{2}$ & $95 \%$ IC & $\begin{array}{c}\text { Prueba } \\
\text { tendencia }\end{array}$ \\
\hline \multicolumn{8}{|l|}{ Edad } \\
\hline$£ 25$ & 51 & 59 & 1,0 & & 1.0 & & \\
\hline $26-35$ & 61 & 118 & 0,59 & $0.36-0.97$ & 0.64 & $0.37-1.10$ & \\
\hline $36-45$ & 29 & 95 & 0.35 & $0.20-0.61$ & 0.36 & $0.19-0.66$ & \\
\hline 346 & 13 & 44 & 0,34 & $0.16-0.70$ & 0.32 & $0.15-0.71$ & 0.05 \\
\hline \multicolumn{8}{|c|}{ Menarquia } \\
\hline $9-11$ & 20 & 37 & 1,0 & & 1.0 & & \\
\hline 12 & 35 & 78 & 0,83 & $0.42-1.62$ & 0.83 & $0.42-1.63$ & \\
\hline 13 & 44 & 84 & 0.96 & $0.50-1.86$ & 0.97 & $0.50-1.88$ & \\
\hline 314 & 55 & 117 & 0,86 & $0.46-1.63$ & 0.87 & $0.46-1.65$ & 0.81 \\
\hline \multicolumn{8}{|c|}{ Edad IVSA } \\
\hline 17 & 72 & 129 & 1,0 & & 1.0 & & \\
\hline $18-20$ & 50 & 110 & 0,81 & $0.52-1.26$ & 0.85 & $0.52-1.39$ & \\
\hline 321 & 32 & 77 & 0,74 & $0.45-1.23$ & 0.81 & $0.41-1.57$ & 0.31 \\
\hline \multicolumn{8}{|c|}{ No. de embarazos } \\
\hline 0 & 6 & 10 & 1,0 & & 1.0 & & \\
\hline $1-2$ & 68 & 106 & 1,06 & $0.37-3.07$ & 2.38 & $0.45-12.5$ & \\
\hline $3-4$ & 45 & 96 & 0.78 & $0.26-2.28$ & 1.81 & $0.38-8.63$ & \\
\hline 35 & 35 & 104 & 0,56 & $0.19-1.65$ & 1.36 & $0.30-6.15$ & 0.23 \\
\hline \multicolumn{8}{|c|}{ No. partos } \\
\hline 0 & 29 & 55 & 1,0 & & 1.0 & & \\
\hline $1-2$ & 58 & 88 & 1,25 & $0.71-2.18$ & 1.35 & $0.64-2.83$ & \\
\hline $3-4$ & 44 & 89 & 0.93 & $0.52-1.66$ & 1.08 & $0.50-2.32$ & \\
\hline 35 & 23 & 84 & 0,52 & $0.27-0.98$ & 0.67 & $0.28-1.60$ & 0.27 \\
\hline \multicolumn{8}{|c|}{ No. de abortos } \\
\hline 0 & 125 & 239 & 1,00 & & & & \\
\hline${ }^{3} 1$ & 29 & 77 & 0,72 & $0.44-1.16$ & 0.88 & $0.52-1.47$ & \\
\hline \multicolumn{8}{|c|}{ No. de cesáreas } \\
\hline 0 & 117 & 246 & 1,00 & & 1.00 & & \\
\hline 1 & 26 & 35 & 1.5 & $0.89-2.71$ & 1.52 & $0.86-2.68$ & \\
\hline 2 & 6 & 24 & 0,52 & $0.20-1.32$ & 0.50 & $0.19-1.29$ & \\
\hline 33 & 5 & 11 & 0,95 & $0.32-2.81$ & 0.94 & $0.31-2.82$ & 0.69 \\
\hline \multicolumn{8}{|c|}{ Edad $1^{\text {er }}$ embarazo } \\
\hline 17 & 53 & 82 & 1,00 & & 1.00 & & \\
\hline $18-22$ & 69 & 160 & 0,66 & $0.42-1.42$ & 0.60 & $0.37-0.98$ & \\
\hline 323 & 26 & 64 & 0,62 & $0.35-1.11$ & 0.49 & $0.25-0.97$ & 0.02 \\
\hline \multicolumn{8}{|c|}{ No. parejas sexuales } \\
\hline 1 & 118 & 248 & 1,00 & & 1.00 & & \\
\hline 2 & 24 & 43 & 1,17 & $0.67-2.02$ & 1.23 & $0.70-2.18$ & \\
\hline 33 & 12 & 25 & 1.00 & $0.48-2.07$ & 1.29 & $0.60-2.75$ & 0.77 \\
\hline
\end{tabular}

$\mathrm{OR}^{1}$ : Odds ratios crudo. $\mathrm{OR}^{2} \mathrm{Odds}$ ratios ajustada por: edad, edad de primer embarazo, número de partos, número de cesáreas y número de parejas sexuales. 
Consideramos que la diferencia inicial que se observó en el análisis univariado en cuanto a partos y gestaciones era dependiente de la edad, por lo cual dicha diferencia desaparece al hacer ajuste de variables de confusión.

El análisis multivariado demuestra una clara tendencia de protección para la IPVH al aumentar la edad de la paciente, resultado que está acorde con lo reportado por otros autores que han abordado el tema y dejan claro que las mujeres menores de 25 años tienen mayor riesgo de infectarse (17-19).

En el análisis de regresión logística se observó un efecto protector del primer embarazo después de los 23 años. Esta asociación no había sido descrita antes y será necesario realizar estudios con una muestra mayor para confirmarla o descartarla.

La estrecha relación entre el número de compañeros sexuales y la IPVH ha sido documentada en países como Estados Unidos, Canadá y Holanda; sin embargo, en los estudios con poblaciones de origen latino los resultados han sido diferentes $(11,12,15,16,17)$. En nuestro estudio no se encontró ninguna relación entre el número de compañeros sexuales y el riesgo de infección por PVH.

Actualmente existen evidencias epidemiológicas que demuestran que los factores de riesgo asociados a los PVH de alto riesgo no son los mismos que los que se asocian a los PVH de bajo riesgo; ello sugiere que el grado de transmisión por vía sexual varía según el tipo de virus $(20,22)$.

En nuestro estudio, el diagnóstico de PVH se realizó por citología y colposcopia, lo cual no nos permite conocer el tipo de virus presente en la población estudiada; esta limitante podría ser la razón por la cual no se encontró asociación entre número de compañeros sexuales y PVH.

Se toman como marco de referencia los resultados obtenidos por otros investigadores que no encuentran asociación entre número de compañeros sexuales y PVH de bajo riesgo.
Existen estudios realizados en mujeres de origen latino que sugieren que existe algún factor que no está relacionado con la historia sexual de las mujeres que las pone en riesgo de infectarse por $\mathrm{PVH}$.

Reeves (23) realizó un estudio con mujeres panameñas que habían tenido sólo un compañero sexual y menos de 15 años de vida sexual; al comparar la prevalencia de VPH16/18 entre las mujeres del área rural y las del área urbana, comprobó una mayor prevalencia en las mujeres del área urbana.

Más recientemente, Giuliano (16) realizó un interesante estudio en mujeres de ascendencia mexicana, unas nacidas en México y otras en Estados Unidos; entre sus resultados relevantes está el hecho de que 63,5\% de las nacidas en México sólo habían tenido un compañero sexual, a diferencia de $34,8 \%$ de las nacidas en Estados Unidos.

Cuando realizó un estudio comparativo entre los dos grupos se observó que las mujeres nacidas en México tenían un perfil de menor riesgo, pero su OR era mayor.

Las mujeres de este estudio eran todas nacida en México y la mayoría proveniente del área rural, culturalmente diferente a las poblaciones en las cuales se ha encontrado una fuerte asociación entre el número de compañeros sexuales y la infección por virus de papiloma, y semejante a las poblaciones estudiadas por Giuliano y Reeves, por lo que consideramos que en este grupo podría existir un factor asociado a sus compañeros sexuales que estuviera jugando un papel importante en la infección del papilomavirus $(16,23)$.

Aunque el presente trabajo tiene limitaciones de índole diagnóstica, consideramos que a la luz de los conocimientos actuales no se deben extrapolar los resultados de investigaciones realizadas en poblaciones culturalmente diferentes.

Así mismo, es importante plantear estudios más completos, con una perspectiva biológica, social y antropológica, en los cuales se tipifique el virus, se 
estudie a los compañeros sexuales de las mujeres y se comparen mujeres de área rural y urbana para poder conocer y comprender los factores que están interviniendo en la transmisión de la infección del PVH en mujeres mexicanas.

\section{REFERENCIAS BIBLIOGRÁFICAS}

1. Parkin DM, Pisani P, Ferlay J. Estimates of worldwide incidence of eighteen major cancers in 1995. Int J Cancer 1993;54:594-606.

2. Robles SC. Tamizaje del cáncer de cuello de útero presentación del número monográfico. Bol Of Sanit Panam 1996;121:473-477.

3. Manual para la vigilancia epidemiológica de cáncer cérvico-uterino. Secretaría de Salud. 1998.

4. Muñoz N, Bosch X. Cervical cancer and human papillomavirus: epidemiological evidence and perspectives for prevention. Sal Púb Méx 1997;39:274282.

5. Bosch FX, Manos, MM, Muñoz, N, et al. The IBSCC study group: Prevalence of human papillomavirus in cervical cancer: A worldwide perspective. J Natl Cancer Inst 1995;87:796-802.

6. Herrero R, Brinton LA, Reeves W, et al. Factores de riesgo de carcinoma invasor y cuello uterino en América Latina. Bol Of Sanit Panam 1990;109:6-22.

7. Schiffman M, Bauer H, Hoover R. Epidemiologic evidences showing that HPV infections causes most cervical intraepitelial neoplasis. J Natl Cancer Inst 1993;85:958-964.

8. Koutsky LA. Epidemiology of genital human papillomavirus infection. Am J Med 1997;102:3-8.

9. Bauer HM, Yiting MS, Greer CE. Genital human papillomavirus infection in females university students as determined by PCR-based method. JAMA 1991;265:472-477.

10. Gravitt PE, Peyton CL, Apple RJ, Wheeler CM. Genotyping of 27 human papillomavirus types using L1 consensus PCR products by single hybridization, reverse line blot detection method. J Clin Microbiol 1998;36:3020-27.
11. Burk RD, Ho GFY, Beardsley L, et al. Sexual behaviour and partner characteristics are the predominant risk factors for genital human papillomavirus infection in young women. J Infect Dis 1996;174:679-689.

12. Kenney JW. Risk factor associated with genital HPV infection. Cancer Nur 1996;19:353-359.

13. Svare EI, Kjaer SK, Smith HL, et al. Risk factors for HPV detection in archival pap smear. A populationbased study from Greeland and Denmark. Eur J Cancer 1998;34:1230-1234.

14.Hernández-Ávila M, Lazcano-Ponce CE, BerumenCampos J, et al. Human papilomavirus 16/18 infection and cervical cancer in Mexico: A case-control study. Archives of Med Res 1997;28:265-271.

15. Reed BD, Zazove P, Gregorie L, et al. Factors associated with human papillomavirus infection in woman encountered in community-based office. Arch Fam Med 1993;2:1239-48.

16. Giuliano AR, Papenfuss M, Scheider A, Nour M, Hatch K. Risk factors for high-risk type human papillomavirus infection among Mexican-American women. Cancer Epidemiol Biomarkers Prev 1999;8:615-620.

17. Svare EI, Kjaer SK, Worm AM, et al. Risk factors for HPV infection in women from sexually transmitted diseases clinics: comparison between two areas with different cervical cancer incidence. Int J Cancer 1998;138:105-115.

18. Figueroa JP, Ward E, Luthi TE, et al. Prevalence of human papillomavirus among STD clinics attainders in Jamaica: association of younger age and increased sexual activity. Sex Trans Dis 1995;22:114-118.

19. Viscidi RP, Clayman B, Russ K, Shapiro S, Shah KV. Prevalence of antibodies to human papillomavirus type 16 virus-like particles in relation to cervical infection among college woman. Clin Diag Lab Immunol 1997;4:122-128.

20.Kjaer SK, Vanden-Brule AJC, Bock JE, et al. Determinants for genital human papillomavirus infection in 1000 randomly chosen young Danish woman with normal papa smear. Are there different risk profiles for oncogenic and nononcogenic HPV types. Cancer Epidemiol Biomarkers Prev 1997;6:799805. 
21. Franco EL, Villa LL, Ruiz A, Costa MC. Transmission of cervical human papillomavirus infection by sexual activity: Differences between low and high oncogenic risk types. J infect Dis 1995;172:756-763.

22. Richardson H Franco E, Pintos J, Bergeron J, Arella M, Tellier P. Determinants of low-risk and high risk cervi- cal human papillomavirus infection in Montreal university students. Sex Transm Dis 2000;27:79-86.

Reeves WC, Gary HE Jr, Johnson PR, et al. Risk factor for genital papillomavirus infection in populations at high and low risk for cervical cancer. J Inf Dis 1994; 170:753-758. 